\title{
"Smart" Surveillance of Dusty Behavior: Illuminating the Relationship between Particulate Matter and the Atmosphere
}

\author{
Shelley Choi \\ Yongsan International School of Seoul, 285 Itaewon-ro, Yongsan-gu, Seoul, South Korea \\ Email: 19.shelley.choi@yisseoul.org
}

How to cite this paper: Choi, S. (2018) "Smart" Surveillance of Dusty Behavior: Illuminating the Relationship between Particulate Matter and the Atmosphere. Computational Water, Energy, and Environmental Engineering, 7, 119-126. https://doi.org/10.4236/cweee.2018.73008

Received: April 12, 2018

Accepted: July 17, 2018

Published: July 20, 2018

Copyright $\odot 2018$ by author and Scientific Research Publishing Inc. This work is licensed under the Creative Commons Attribution International License (CC BY 4.0).

http://creativecommons.org/licenses/by/4.0/

\begin{abstract}
Although large amounts of research have been completed to find the relationship between particulate matter and climate change, they have still proven to be inadequate. Efforts to lay the foundations for understanding atmospheric chemical reactions have been repeatedly foiled by both the size and complexity of the task, which require more than the effort of a handful of researchers. Since the development of advanced physical models for dust behavior is projected to take years, what if laypeople could dramatically expedite this process by using their mobile devices as measurement tools? With relatively little effort by many individuals, previously unknown information about the earth's atmosphere may at last become accessible thanks to recent advances in artificial intelligence. However, there are potential obstacles. Even if all technical problems are resolved, viable plans for battling particulate matter pollution will likely need to be accompanied by environmental policies. While technological breakthroughs give reason to hope for a brighter future, the resolution of global issues requires both grassroots changes and global efforts.
\end{abstract}

\section{Keywords}

Atmosphere, Particulate Matter, Weather Forecast, Artificial Intelligence, Deep Learning

\section{Introduction}

To this day, the atmosphere remains a more or less inviolable province. We are mere observers to this domain; our understanding of the chemical interactions occurring within it is cursory at best. The scientific community has recently begun to devote an increasing amount of attention to the relationship between dust and climate change: "Understanding the links between dust and climate in the 
past will be crucial to evaluate the future impacts of dust on the Earth's climate system in a warming world" [1]. Despite some hurdles, strides taken in the development of both artificial intelligence and machine learning will help illuminate the science behind atmospheric chemistry and equip humanity to reliably explain, predict, and reduce air pollution and climate change. Even so, the tools themselves could prove to be insufficient agents of change. As history tells us, real change can only occur when concerned citizens, institutions, and government work together towards a common goal.

\section{Particulate Matter and Its Behavior}

The wildfire scourge that ravaged the western United States in September 2017 brought about unprecedented levels of destruction on the region and filled the air with dangerous levels of pollution. The air pollution that choked these states forced residents at otherwise safe distances from the wildfire to flee their homes, schools, and workplaces. However, what was less reported were the far-reaching, global consequences caused by a local, seemingly isolated, event. Once the haze retreats, the residents will return to their lives and carry on business as usual. Not many of them think of the early September blaze as they enter the especially soggy winter months. Although the haze will dissipate, the particles that caused it will become dispersed throughout the earth's atmosphere, altering its chemical composition and adding to climate change for years to come.

Because the atmosphere is a natural crossroad where the products of reactions from around the world interact, this idea should not sound too far-fetched. The classic study done by the North Atlantic Aerosols and Marine Ecosystems Study (NAAMES) found a strong correlation between carbon and marine plankton populations. In particular, phytoplankton in the oceans produce dimethyl sulfide, which becomes rapidly oxidized to sulfate upon entering the atmosphere, generating what is called sulfate aerosols. When sulfate aerosols bind with water vapor, clouds are formed through condensation. On the one hand, because "plankton are an important carbon sink for atmospheric greenhouse gases," the increased availability of carbon from both natural and manmade sources leads to a surge in phytoplankton population, and then to a colder and wetter climate [2]. On the other, the warmer the ocean, the less the carbon it harbors, limiting the growth of phytoplankton. Fewer plankton mean less cloud to cool the earth, hence warmer surface temperatures (Figure 1).

Such a finding is only part of a bigger picture as more data is becoming available to both complement and complicate our grasp of atmospheric chemistry. For example, the particulate matter emitted during the high-temperature combustion of biomass during the wildfires contains brown carbon, which rises through the atmosphere because its formations are less dense than black carbon's formations. These brown carbon formations absorb and scatter solar radiation, cooling the earth's surface [2]. Yet even as of 2017, particulate matter remained poorly defined and the definition itself included a wide variety of sizes and 


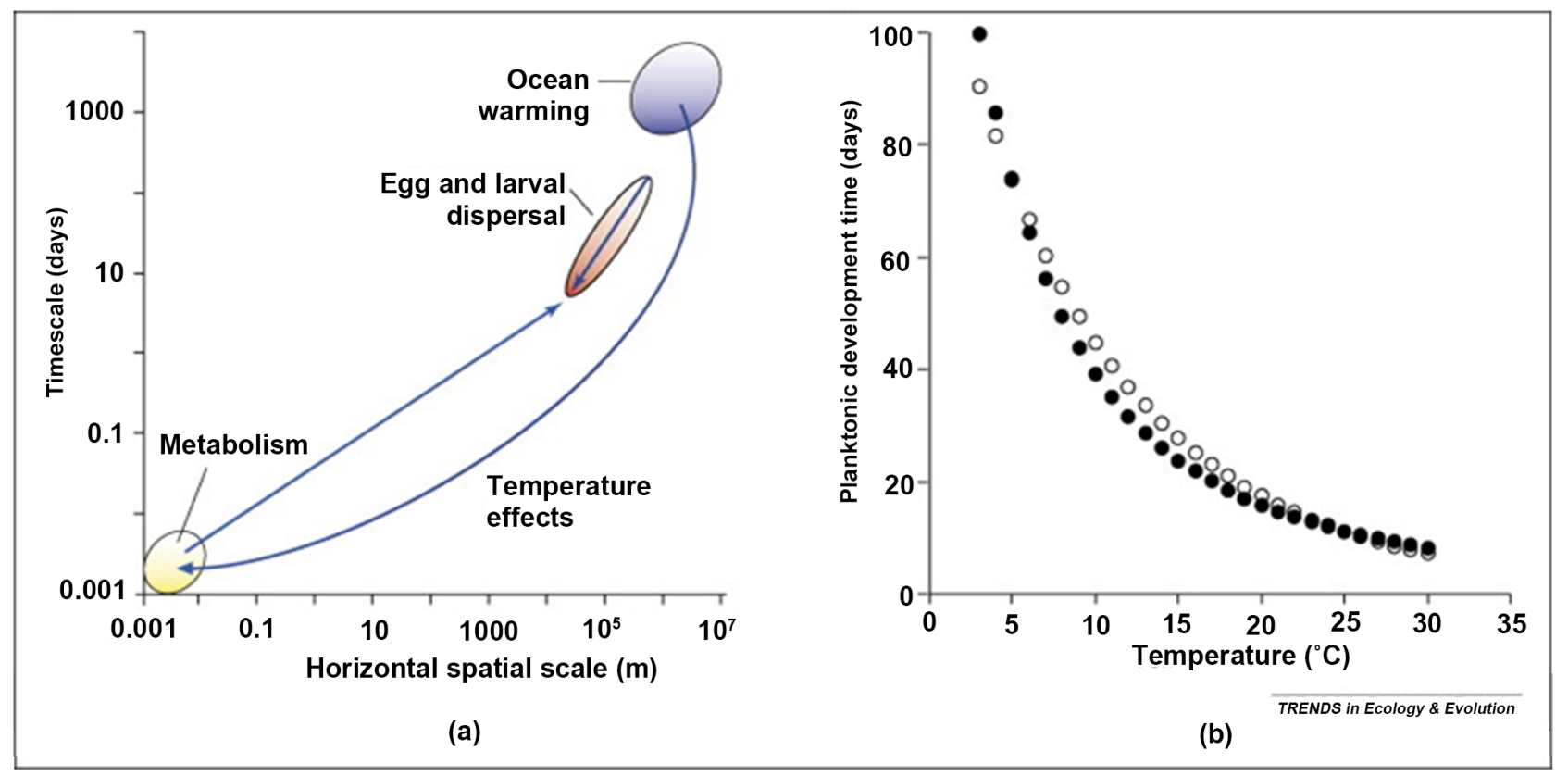

Figure 1. Effects of temperature and ocean warming on planktonic egg and larval development times and associated dispersal distances (p. 332) [3].

configurations. It could be as small as a single molecule, or as large as a 10-micron dust. Kok et al. (2011) found that 1- to 5-micron particles contributed the most to atmospheric fluctuations [4]. Based on their findings, a great deal of emphasis today is placed on making distinctions between fine $(<2.5$ microns $)$ and coarse (2.5 - 10 microns) particles. While fine particles interact with sunlight to lower the temperature of the air by acting like tiny mirrors, coarse particles raise the temperature of the air by absorbing heat. Although particle size is significant, it is not the only attribute that matters. This year, Kok et al. concluded that an addition of $2000 \mathrm{Tg}$ of particles per year released into the atmosphere can make a tangible impact on the climate [4]. The study found that, while the same amount of dust generated over a short period may have similar effects, it is not comparable to that of the same amount accumulated at a more constant rate over the span of a year. Given that particles of any size may combine to form even larger particles, these factors may be irrelevant [5]. Thus, it is just as important to study particle behavior as it is to study the particles themselves. According to Pospisil and Jicha, such behavior is "a very complex process [that is difficult to describe mathematically]" (p. 157) [6]. The notable degree of entropy and lack of linearity in dust behavior contribute to this difficulty, as well as the lack of useable data.

Dust pollution is a daily reality familiar to many people living in industrial societies. Climate researchers have just begun to suspect that climate change triggered by microscopic dust could be likened to harm to the people. In fact, particulate matters can irritate the eyes, nose, and throat, and cause respiratory and cardiovascular diseases (Figure 2). 
Studying the effects of these particulates in a vacuum may appear simple, but the earth's atmosphere is a wide-open arena of myriad aerodynamic vectors that interact with each other in varied and unpredictable ways. The popular regional and global circulation model tracks airborne circulation by dividing the atmosphere and oceans into 40 - 50 vertical layers of $250 \mathrm{~km}^{2}$ units [8]. However, the coarseness of the model's division of the atmosphere means that its predictions are relatively inaccurate, especially for matter smaller than 2 microns. Kok found gross inaccuracies in an analysis of clay aerosols, the radiative cooling effect of which is of significant interest in the field [9]. The global circulation model "overestimate $[\mathrm{d}$ ] the emitted fraction of clay aerosols $(<2 \mu \mathrm{m}$ diameter $)$ by a factor of $\sim 2$ - 8 relative to measurements" (p. 1016). As this example shows, sufficiently accurate advanced physical models of dust behavior are projected to take years to develop. Given the immensity of the Earth's surface, the research required to develop such models is sure to involve more researches. At the same time, the Earth is already undergoing drastic climate change, so the need to accelerate research is more pressing than ever.

\section{Grassroots Change at Fingertips}

Even if the technical challenges surrounding particulate matter behavior could be addressed, coming up with a viable solution for battling dust pollution on national and international levels will likely take the difficult path of clearing legislative, financial, and diplomatic gridlocks. Environmental policy must keep in step with environmental changes, and the responsibility to spearhead environmental activism falls on the well-informed, as raising awareness is at the beginning of any large-scale movement. In fact, the role of information amounts

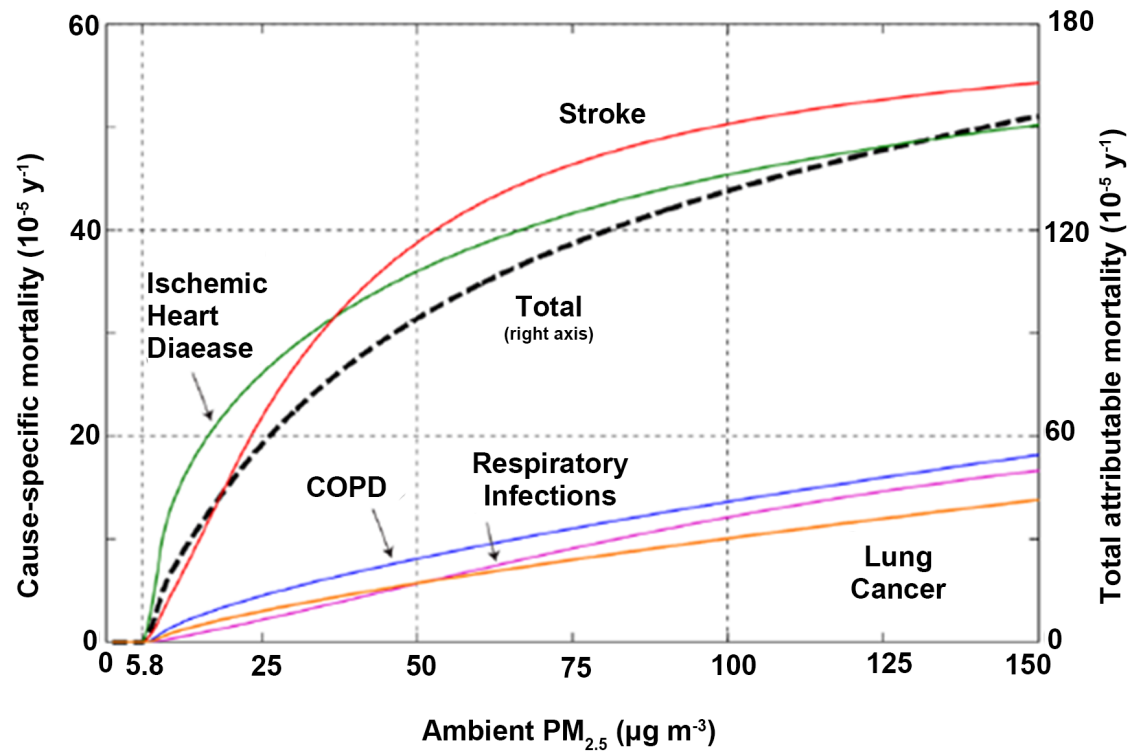

Figure 2. Global concentration-mortality relationships for ambient $\mathrm{PM}_{2.5}$ for five individual endpoints and total of five causes (p. 8058) [7]. 
to more than just spreading relevant data. As an example, one may look to the immense influence exerted by Rachel Carson's Book Silent Spring on the establishment of the Environmental Protection Agency in December 1970. As acknowledged by the Agency itself, Silent Spring "played in the history of environmentalism roughly the same role that Uncle Tom's Cabin played in the abolitionist movement. In fact, EPA today may be said without exaggeration to be the extended shadow of Rachel Carson" (p. 6) [10]. What Carson did to lead the Environmental Movement of the late 1960's to such success was not only due to her exhaustive research related to environmental destruction and its effects on humans, but also due to her revelatory argumentation. By weaving seemingly unrelated incidents and facts into a coherent, science-based narrative, Carson allowed the public to easily grasp the state of affairs while urging them towards action. Though it is not to say that activism is impossible without the help of intellectuals, their role in promoting awareness may be overwhelming.

What if, then, laypeople could also empower the process of measuring, analyzing, evaluating, and proposing solutions for the particulate matter debacle as well? What if they could use their mobile devices as measurement tools? Existing communications technology and innovations in artificial intelligence might reduce development time. Every smartphone user can install a measurement application on their smartphones: measurement devices are compact and can even be embedded in headphones or personal Bluetooth devices [11]. The measurement application can run in the background, just like a music streaming application, continuously taking readings of the surrounding air, including information such as the density of airborne dust, fumes, temperature, wind speed, and humidity [12]. One application could provide visualization and other analytical functionalities, which helps to educate users on their environments and eases computational bottlenecks at data centers by performing processing on users' devices. The same devices could be installed on building surfaces or rooftops to collect data from higher altitudes. Personal drones and other aerial vehicles such as helicopters can also play a vital role in collecting high-altitude data. This solution does not require people to make drastic departures from their current lifestyles and does not require the completion of a large-scale project devoted to a single purpose. Most importantly, such a system makes it possible to take atmospheric measurements anytime, anywhere, which was previously nearly impossible.

\section{How Machine Learning Can Help}

The data, as noted above, only makes up half of the equation: the other half is developing effective mathematical models using machine learning. Modeling complex systems such as the atmosphere present substantial challenges. Because there are so many variables that influence the model's output, establishing the relationships between each variable to make accurate predictions is nearly impossible. This type of problem is referred to as complex function approximation because an association between a set of observed variables and a prediction is a 
function in a mathematical sense. Traditionally, function approximation problems were first approached by assuming that the model would be accurately represented by a particular mathematical function, such as a linear function or a polynomial of a particular order. Any mathematical function can be described in terms of its type and a set of parameters. In this context, this model choice refers to the type of function used to make computations regarding the atmosphere. It naturally reflects a set of assumptions made about the nature of the input variables and the structure of the system that is being modeled. These assumptions are essentially pre-hoc commitments made by scientists that are somewhat independent from the data.

For example, in order to make predictions about the movement of a spring, a linear spring equation would serve as an adequate model. They make hundreds of measurements by manipulating the spring to estimate the spring equation's parameters. The spring equation is a "good model" not because it is more accurate than others, but because it is the simplest known expression which sufficiently and accurately represents the movement of the spring within a reasonable range of circumstances. That is, for any given model, there are a number of more complex models with an appropriate set of parameters that can explain the data as good or better than the model in question. An example of an alternative model is the model of the weak electric forces exerted by the atoms that make up the spring. The trade-off of this model is that it is more complex with more parameters, which require much more data to fine-tune. Trying to fit a complex model with a small amount of data results in over fitting, a phenomenon in which the model explains the data but is not meaningfully generalizable [13]. Thus, simpler models are usually preferred to more complex models.

This is where machine learning can be used as a time- and cost-saving solution. Machine learning techniques such as deep learning do not require that researchers commit themselves to any particular model before analyzing data that they collect. Deep learning has made the model creation process as simple as feeding data into an algorithm and getting a model in return, making it conceivably practical to predict dust behavior based on data [14]. One field in which deep learning has been used to generate complex models is visual recognition. Developing visual recognition models is a surprisingly similar type of problem to developing weather forecasting models since both phenomena are highly complex with an immense number of variables that are difficult for humans to model. Nonetheless, humans process visual information effortlessly. Deep learning models have revolutionized computer visual recognition and artificial intelligence by allowing computers to develop visual recognition capabilities at a level that many scientists thought would not be seen for another century, so the power of deep learning can be harnessed to solve the problem of model choice. Instead of applying a specific model, researchers can simply commit to the number of total parameters that the algorithm can analyze and the dimensions of a neural network model, which is a conceptual embodiment of the mathematical model 
employed by deep learning. Then the deep learning algorithm analyzes the data and determines which parameters are not particularly helpful and removes them from the model while tuning the remaining parameters [15].

\section{Conclusion}

This technology will help researchers make accurate predictions and generalizations based on collected atmospheric data, and the wealth of new data will create exciting new avenues for research in fields of natural sciences and engineering. Such a concerted effort will not only aid in addressing the dust epidemic, but it will also take weather forecasting techniques to a new level by allowing meteorologists to predict extreme weather changes more accurately [16]. However, potential obstacles do exist. Although involving large portions of the global population would be an unparalleled advantage, the asymmetry in population densities at different geographic locations and altitudes and the creation of false data may initially yield a skewed picture of atmospheric conditions. Even if all technical problems are resolved, viable plans for actually battling particulate matter pollution would likely need to meet with legislative approval and be provided with financial resources. Just like any other policy, the best environmental policy is one which is initiated by the public, fully utilizes available democratic apparatus, and is keenly watched over by the larger public. Recent experience indicates that the challenge should not be taken lightly because broadly effective action on combatting climate change cannot happen solely at the grassroots level; the resolution of global crises requires global efforts.

\section{References}

[1] Winckler, G. (2010) Dust and Its Impact on Earth's Climate System. State of the Planet.

http://blogs.ei.columbia.edu/2010/06/17/dust-and-its-impact-on-earth\%E2\%80\%99 s-climate-system/

[2] Zhang, Y., Forrister, H., Liu, J., Dibb, J., Anderson, B., Schwarz, J.P., Weber, R.J., et al. (2017) Top-of-Atmosphere Radiative Forcing Affected by Brown Carbon in the Upper Troposphere. Nature Geoscience, 10, 486-489.

https://doi.org/10.1038/ngeo2960

[3] Duarte, C.M. (2007) Marine Ecology Warms up to Theory. Trends in Ecology and Evolution, 22, 331-333. https://doi.org/10.1016/j.tree.2007.04.001

[4] Kok, J.F., Ridley, D.A., Zhou, Q., Miller, R.L., Zhao, C., Heald, C.L. and Haustein, K. (2017) Smaller Desert Dust Cooling Effect Estimated from Analysis of Dust Size and Abundance. Nature Geoscience, 10, 274-278. https://doi.org/10.1038/ngeo2912

[5] Green Facts (2005) Air Pollution Particulate Matter. https://www.greenfacts.org/en/particulate-matter-pm/

[6] Pospisil, J. and Jicha, M. (2008) Behavior of Particulate Matter Produced by Cars in a Regional Model of Urban Canopy Layer. Transactions on Transport Sciences, 1, 157-164. https://doi.org/10.5507/tots.2008.021

[7] Apte, J.S., Marshall, J.D., Cohen, A.J. and Brauer, M. (2015) Addressing Global Mortality from Ambient $\mathrm{PM}_{2.5}$. Environmental Science and Technology, 49, 
8057-8066. https://doi.org/10.1021/acs.est.5b01236

[8] Pitman, A.J., Henderson-Sellers, A. and Yang, Z.-L. (1990) Sensitivity of Regional Climates to Localized Precipitation in Global Models. Nature, 346, 734-737. https://doi.org/10.1038/346734a0

[9] Kok, J.F. (2010) A Scaling Theory for the Size Distribution of Emitted Dust Aerosols Suggests Climate Models Underestimate the Size of the Global Dust Cycle. Proceedings of the National Academy of Sciences of the United States of America (PNAS), 108, 1016-1021. https://doi.org/10.1073/pnas.1014798108

[10] Lewis, J. (1985) The Birth of EPA. EPA Journal, 11, 6-11.

[11] Casella (n.d.).

http://www.casellasolutions.com/us/en/products/dust-and-gases/hand-held/produc ts/microdust-pro.aspx

[12] Chen, J.X. and Fu, X. (1999) Integrating Physics-Based Computing and Visualization: Modeling Dust Behavior. Computing in Science and Engineering, 1, 12-16. https://doi.org/10.1109/5992.743611

[13] Cai, E. (2014) Machine Learning Lesson of the Day-Overfitting and Underfitting. http://www.statsblogs.com/2014/03/20/machine-learning-lesson-of-the-day-overfitt ing-and-underfitting

[14] Aherne, C. (2017) How Machine Learning Can Be Used to Predict Customer Behaviour.

https://www.altocloud.com/blog/how-machine-learning-can-be-used-to-predict-cu stomer-behaviour

[15] Sestili, C. (2018) Deep Learning: Going Deeper toward Meaningful Patterns in Complex Data.

https://insights.sei.cmu.edu/sei_blog/2018/02/deep-learning-going-deeper-towardmeaningful-patterns-in-complex-data.html

[16] Liu, Y., Racah, E., Prabhat, Correa, J., Khosrowshahi, A., Lavers, D. and Collins, W. (2016) Application of Deep Convolutional Neural Networks for Detecting Extreme Weather in Climate Datasets. In: Proceeding from ABDA'16: The $3 r$ International Conference on Advances in Big Data Analytics, CSREA, Las Vegas, 81-88. 